\title{
Improving the Introduction to a Collaborative Project- Based Course on Computer Network Applications
}

\author{
Felix Freitag $^{1}$, Leandro Navarro ${ }^{1}$, and Joan Manuel Marquès ${ }^{2}$ \\ ${ }^{1}$ Computer Architecture Department, Polytechnic University of Catalonia, Spain \\ ${ }^{2}$ Open University of Catalonia, Spain \\ \{felix, leandro\}@ac.upc.edu, jmarquesp@uoc.edu
}

\begin{abstract}
In engineering studies, there is a shift to new teaching methodologies with focus on the student involvement, like project-based learning. Projectbased learning courses, however, often relay on a previous course where the technical background to be used in the projects is taught, requiring this way two terms for an area. In this paper, we consider a project-based course on computer network applications, which has been designed to cover both the technical and non-technical content in only one term. In the three years we have been teaching this course, our observation based on questionnaires is that the organization of the course allows to successfully reaching the course objectives. We feel, however, that we do not fully exploit the learning potential the course could have in the first few weeks of the course. We describe how the course is organized and the problems we have identified. We propose a project demonstration tool and describe how our solution improves towards our goals. With the proposed tool, the students should better obtain already in the very first days of the course a clear vision about the projects, allowing fully taking advantage of the opportunities which the course offers. format.
\end{abstract}

Keywords: Project-based learning, Course content, Tools for teaching.

\section{Introduction}

In 2003, the computer science curriculum of the Computer Science Faculty of Barcelona (FIB) of the Technical University of Catalonia (UPC) in Barcelona was revised. This revision stated that students of the computer science studies should acquire more non-technical competences (like working in groups, the capacity to manage projects, the oral and written presentation of work, the capacity to learn independently a new technical context and be able to solve new problems).

In order to acquire these new non-technical competences, a number of new courses based on projects were added to the computer science curriculum.

In this paper, we consider one of such project-based learning courses, the course "Project on Computer Network Applications" 1 at the Computer Science Faculty of Barcelona of the Technical University of Catalonia. Courses on Computer Network Applications have recently gained substantial importance in many computer science curricula, being now often part of the core subjects. 
We explain how the course "Project on Computer Network Applications" is organized in order to highlight the improvement we have identified. After three years of teaching experience in this course, our observation based on questionnaires is that both the technical and non-technical objectives of the course are successfully achieved. However, from students' feedback obtained we feel that we do not fully exploit the learning potential the course could offer to the students in the first weeks. We describe the potential for enhancement we have identified and explain our solution. Our solution should provide the students already in the first days a clear vision about the project in the course, which allows better taking advantage of the opportunities offered by the course.

The rest of this paper is organized as follows. In Sec. 2 we explain how the course is organized and identify the problem. In Sec. 3 we evaluate possible solutions. Section 4 explains the design and implementation of our solution, and discusses the expected improvement. Finally, Sec. 5 presents our conclusions.

\section{Problem Identification}

\subsection{Objectives of the Course}

The course "Project on Computer Network Applications" in the Computer Science Curriculum at the Computer Science Faculty of Barcelona of the Technical University of Catalonia has the following objectives, ordered into technical and non-technical objectives [2]:

\section{Technical objectives}

- Choose the appropriate protocol and format for a certain application.

- Design and configure application components and services.

- Define and extend elements of an application to provide services taking into account interoperation, performance, scalability and urity.

- Install and deploy applications necessary for a certain organization.

Non-technical objectives

- Collaborative work 3, oral and written communication, work planning, capability to find information, being able to evaluate alternatives, being able to defend a project.

Furthermore, the course is expected to have 5 ECTS credits [4], which corresponds to a total dedication of $125 \mathrm{~h}-150 \mathrm{~h}$ of the students.

\subsection{Design of the Course}

The technical context in which the project of this course is to be carried out, computer network applications, is new to the students, since in the previous courses on computer networks up to the network level is taught, but the application level is not addressed. Within the technical context of computer network applications, the development of a complete project in group is the main target of our course. 
Taking into account this situation, the course is organized to introduce application layer technologies in the first part of the course to provide the basis by means of small applications developed in laboratory sessions. While these fundamental technologies are becoming known to the student, project groups are formed which choose a project idea and develop a project proposal. The project proposal is prepared in parallel to the laboratory sessions and finishes when the last laboratory sessions also finish. The proposed project is then developed in the group in the second part of the course.

Fig. 1 shows the organization of the course. The first six weeks of the course referring to part I include the laboratory sessions, a few session on theory, and the project proposal. Weeks seven to fifteen, referring to part II, is dedicated to the project development.

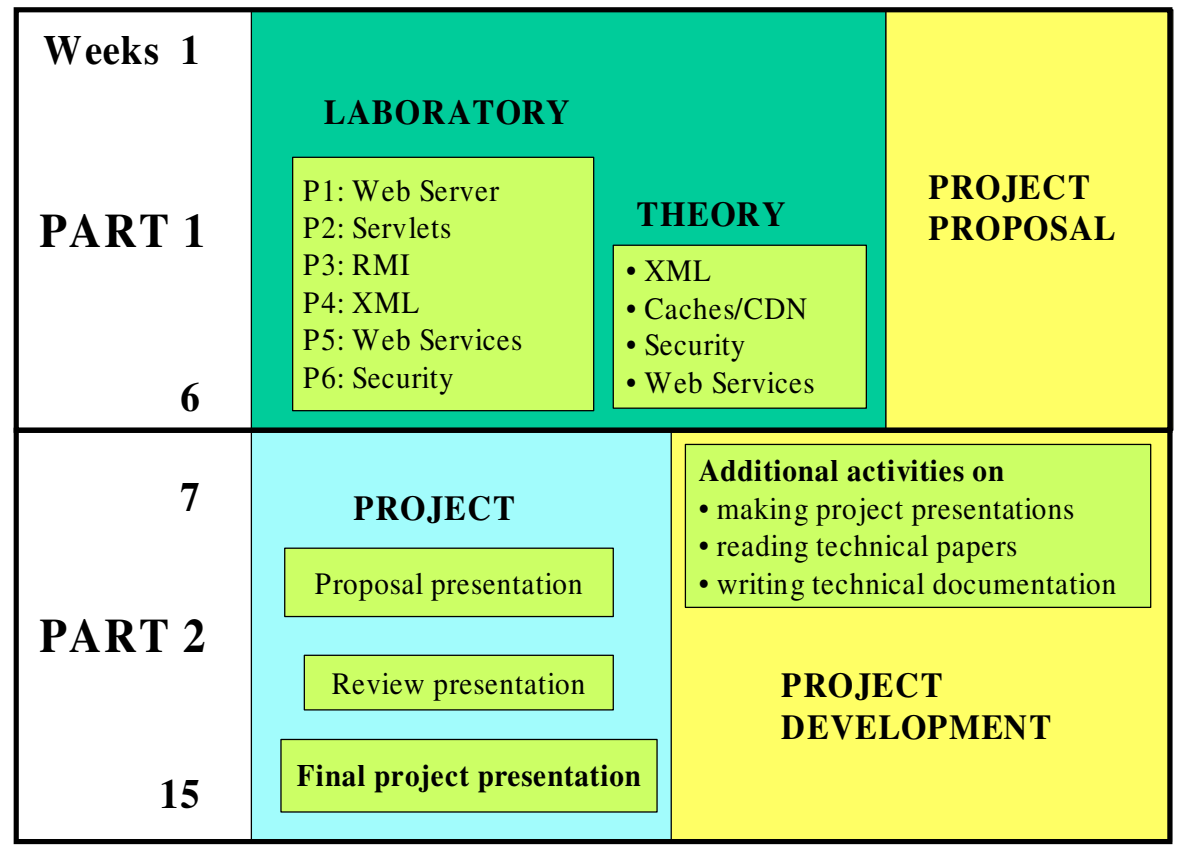

Fig. 1. Schema of the organization of the course

In order to better explain the potential for improvement which we have identified, we describe in the following more details on both parts of the course.

Course organization in the first 6 weeks

The first six weeks of the course focus on the technical foundations of application layer networks. In the first week, groups of two students are formed for the laboratory sessions. Six laboratory sessions are carried out where different application layer technologies are explored by means of simple implementations. At week three, groups of four students are formed as project groups. A project - different for each group but within the technical context of the course - is proposed at week five by the group by means of a written proposal and approved by the lecturer. This proposal includes, 
among other points, the division of the project tasks into work packages and assigning them to members of the group. A Gantt chart is made to define the temporal distribution of the tasks.

\section{Course organization in weeks 7 to 15}

The second part of the course (part II in weeks 7-15) is dedicated to carry out the proposed projects in groups. During this time the projects are developed. In order to achieve the non-technical objectives of the course (see Sec. 2.1), a number of project presentations are scheduled, periodic review meetings with the lecturer take place, project planning and coordination is done, decisions are taken, a demonstration of the project is run, a project presentation to the students is done, and a written project report is elaborated. Optionally, students can realize additional activities concerning how to make the presentation of the project, and how to read and write technical documentation.

\subsection{Problem Statement}

In order to evaluate the course, we have been carrying out a questionnaire via Web with the students at the end of each term, which provides us with feedback from the students' point of view. In order to get the point of view of the lecturers who teach the course, meetings between the lecturers have been taken place.

The information gathered has led to the identification of the following three main problems:

1. Insufficient knowledge of the technologies at the beginning of the course:

Recalling the situation, different to other project-based learning courses, our course does not have a previous course in the computer science curriculum, which provides the technical background on computer network applications. In the project proposal, due at week 5, this lack of sufficiently mature knowledge makes it difficult to estimate the technologies' impact and usage correctly.

2. In the first few weeks, a lack of a clear vision of what is the project in this course: The project proposal is made at week 5 without having seen other similar projects. The project proposal has to be made without being supported by this knowledge.

3. In the first few weeks, the expected results of the project are still not clear: At the time of the project proposal writing, students face the lack of illustrative examples of finished projects, which could allow them assessing better what their project should achieve. Currently, students make the project proposal at week 5 without having this information fully clear.

\section{Our Solution}

The problems identified indicate the need for more information in the first few weeks of the course concerning what is a project in the context of this course. It is clear, however, that a solution to this problem cannot be sought by increasing the number of hours of the course, since the student dedication to the course is already fixed and given by the ETCS credits. 
In order to provide the project knowledge without increasing the course hours, two alternative solutions have been identified:

Alternative 1. Organize the laboratories in part I of the course as a project.

Alternative 2. Develop a project demonstration tool available at the beginning of the course.

Alternative 1, organize the laboratories in the first part of the course as a project, could be reasonable in the sense that a many project proposal include these technologies to implement different components. A particular context like in a project could be sought and used in the laboratories, where in each of the six laboratory sessions a component of the project is then implemented. The result would be that at the end of part I (when at week 6 the laboratory sessions finishes), these sessions have been carried out within the context of a project. This way, all students made a project before developing their own project from week 7 to 15 .

Alternative 2, providing a project demonstration tool to the students, is based on the idea that already in the very first day of the course, information about projects would be made available by such a tool. This tool could support and provide more details about the information on projects given by the lecturer in the first sessions of the course.

Between these alternatives providing a solution to the problem, our choice has been the alternative 2 . The main reason for deciding to develop a project demonstration tool is the early availability of the project information to the students. Already in the first days of the course, illustrative project information can be accessed. On the contrary, alternative 1 would provide a more practical project experience, however the full experience would be available after finishing the laboratories in week 6 , while the project proposal has to be written by the students already in weeks 3 to 5 .

\section{Design and Implementation}

\subsection{User Interface}

The project demonstration tool has been decided to be a web application. The user accesses via a web browser the application server, which provides by means of the GUI different types of project information.

Referring to the content of the user interface, the problems identified in Sec. 2.3 needs to be addressed. This means, the project application tool needs to provide information about

1. technologies for computer network applications,

2. provide a clear vision about what is a project in this course,

3. allow getting a clear picture about the results to be achieved by a project.

Fig. 2 shows a schema of the organization of the main page of the project demonstration tool. The highlighted frames illustrate the access provided to the three main information needs in the main page. In the frames, links to other pages addressing the particular issue are given. 


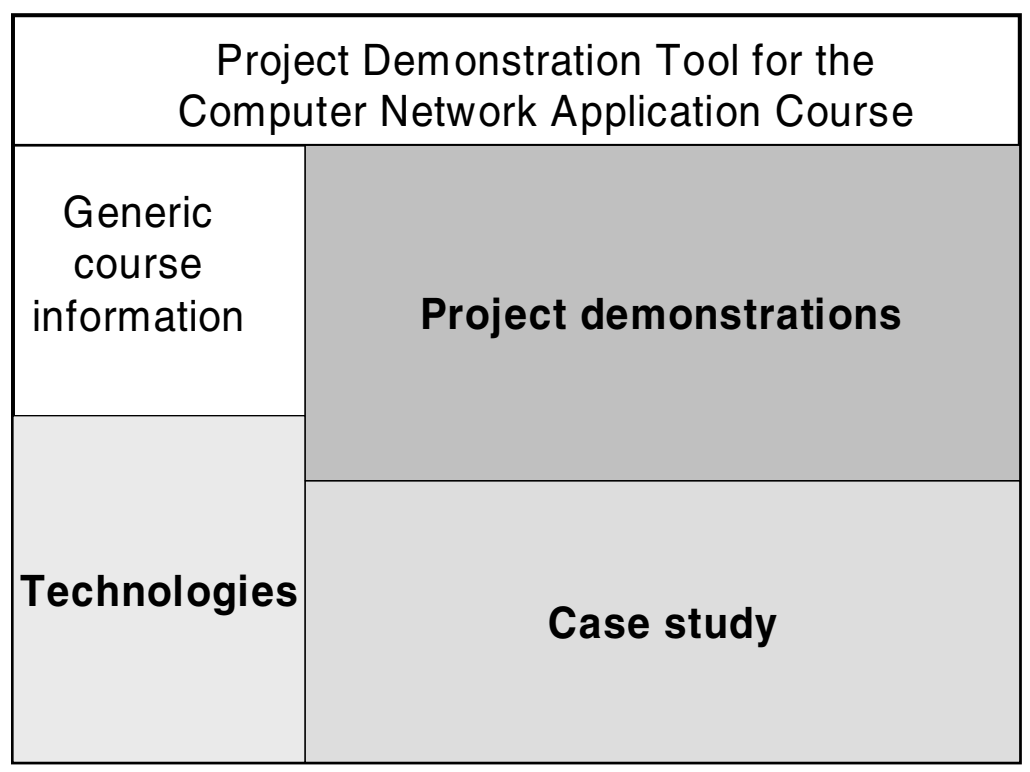

Fig. 2. Organization of main page of the project demonstration tool

The field on technologies allows accessing definitions and use cases of technologies usually applied in any of the projects developed in this course. Depending on the particular technology, information on software packages and comparisons with related technologies is also given. Currently, information about the following technologies is provided: Bluetooth, Peer-to-Peer, J2ME for mobile clients, Ajax, Streaming, the Spring Framework, Apache Struts, and Hibernate.

In the field on project demonstrations, access to a number of project demonstrations in terms of results of applications developed within real projects is provided. These demonstrations are given as videos showing the functioning of the different components of a project, which new students of the course can download. Additionally, code developed within projects can be downloaded for installation, which allows the students to personally executing the applications.

In the case study field, a link to a detailed project execution example is provided. The case study shows by means of a concrete project example how a project is carried out along the whole course. It shows how a project has been envisioned as first ideas and how this leaded to a project proposal. The access to documents generated during the project execution allows showing the progress during the project development and the final result obtained. Perceiving the whole project development in detail allows the students to better perceiving what a project is in the context of this course.

\subsection{Architecture and Implementation}

It was decided to provide the project demonstration tool as a web application. Between different alternatives for the implementation of such an application, like allow advanced support by Content Management Systems, taking advantage of Wiki 
platforms, taking into account the requirement of easy maintenance it was needed to use well known technologies. Therefore, it was chosen to use Apache Tomcat as web server, generate the presentation of the application by JSPs, use servlets for processing the user requests, and use MySQL as the data base.

Fig. 3 shows the architecture of the application. Users are the students who use the application and the administrator. A PC hosts the application server (Apache Tomcat) and the database (MySQL). By design an external FTP server can be used where the videos of the project demos are stored. The FTP server is implemented by means of the ProFTPd tool.

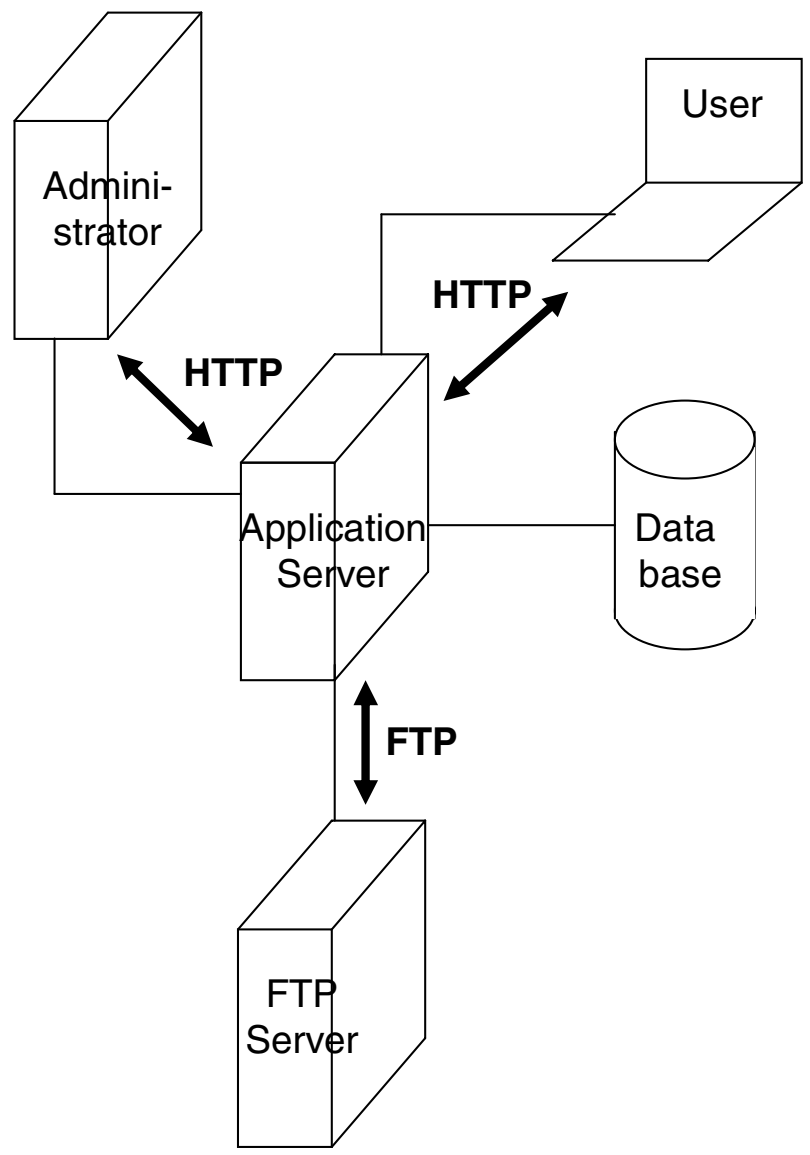

Fig. 3. Architecture

\subsection{Prototype and Evaluation}

The work plan for the development of the project demonstration tool is divided into two phases.

The first phase started in September 2007. At time of this writing, the user interfaces have been defined and the application has been designed. Three projects 
have been selected as demonstrators of finished projects. The description of the technologies they use has been made. The implementation of the first prototype has been completed. The prototype has been deployed in January 2008 on a PC with publicly available IP.

The second phase started in February 2008, at the time when the new course started. The deployed prototype is available to the new students since the beginning of this new course. This second phase focuses first on the feedback gathering from the students and then on a final version of the application which incorporates the suggested improvements.

In order to evaluate the usefulness of the application, the following methods are pursued.

1. Interviews: Informal conversation between the lecturers and the students during the project definition phase in the first weeks of the course will provide data on the intensity of use of the application and the usefulness perceived by the students.

2. Enquiry: During the course (after the project definition phase), an anonymous enquiry via web will be launched to formally capture student's feedback on different aspects of the application.

Concerning the first method, the students who already used the application indicated its usefulness and suggested to add more project demos. On the other hand, we observed that not all students have accessed the application in the first days of the course, which might be related to the fact that the project proposal is due still some six weeks ahead.

\subsection{Discussion on Impact}

The success of the project demonstration tool should be measured by the following impacts:

1. By providing definitions and use cases of the technologies to be used in the projects, it represents an additional source of information to the students that will allow making technically sound project proposals already in the first weeks of the course. It enhances the current situation in which the technologies are shown relatively late and mainly in the context of the laboratories of the course.

2. The demonstration of finished projects allows the students to make a better estimation of the project goals and the related efforts. This will allow making more accurate project proposals.

3. A detailed example of a project execution both contributes the students to better elaborate the management part of the project in the project proposal, and to actually carry out this management during the project execution.

\subsection{Vision on Future Deployment}

Considering the need to store a growing number of video demos as suggested by the students, we will need to add dynamically additional storage capabilities to those currently available in the PC which hosts the FTP server. We consider therefore 
adapting the hosting of the videos to the Grid storage infrastructure (VOFS) which is currently developed within the Grid4All project [5]. This way, the FTP server will be able to dynamically increase its storage resources if needed. It could therefore adapt to a sudden increase of video demos which might happen by the end of the course when a large number of student projects finishes. Another aspect of integrating part of the application in Grid4All which is interesting for the course from an instructor point of view is that the application itself could become a demonstrator of a Grid enabled application.

\section{Conclusions}

The paper showed by means of a project-based computer network applications course, that such courses might not fully take advantage of the learning potential available in the first few weeks of the course, due to not providing students with sufficient practical and illustrative examples of projects. Revising the organization of this course, the benefits of such project information at the beginning of the course were stated.

A project demonstration tool was chosen as a solution to support the lecturer in teaching at the beginning of the course on project related issues. It was described how the project demonstration tool could address the current lack of practical information. The information provided will allow the student acquiring more knowledge in the first weeks of the course, allowing them to better exploit the course's opportunities.

The course presented, project-based computer network applications, achieved teaching both the technical and non-technical contents in one term. This experience could interesting in the sense that other project-based course rely on the technical background provided in another previous course. Our experience could motivate to think if other project-based courses could also be enabled to cover an area in one term instead of two in the curriculum.

Acknowledgements. This work has been partially financed by the Grid4All European project under contract FP6-IST-034567, the Spanish Ministry of Education and Science under contract TIN2007-68050-C03-01, FEDER funds, and the Technical University of Catalonia.

\section{References}

1. Course Project on Computer Networks at Computer Science Faculty at UPC Barcelona, http: / /www. fib.upc. edu/en/infoAca/estudis/assignatures/PXC.html

2. CRITERIA FOR ACCREDITING COMPUTING PROGRAMS. Accreditation Board for Engineering and Technology (ABET), http: / / www . abet .org

3. Johnson, D.W., Johnson, R.T., Smith, K.A.: Active Learning: Cooperation in the College Classroom (1998)

4. European Credit Transfer System, http://europa.eu.int/comm/education/ socrates/ects.html

5. Grid4all project: http://grid4all.elibel.tm.fr/ 